\title{
Réflexions sur les termes-valises en anglais de l'économie, de la gestion et de la finance
}

Reflections on blends in English for economics, management and finance

\section{Catherine Resche}

\section{(2) OpenEdition}

1 Journals

Édition électronique

URL : http://journals.openedition.org/asp/4709

DOI : 10.4000/asp.4709

ISSN : 2108-6354

Éditeur

Groupe d'étude et de recherche en anglais de spécialité

\section{Édition imprimée}

Date de publication : 23 octobre 2015

Pagination : $67-85$

ISSN : 1246-8185

\section{Référence électronique}

Catherine Resche, «Réflexions sur les termes-valises en anglais de l'économie, de la gestion et de la finance », ASp [En ligne], 68 | 2015, mis en ligne le 01 novembre 2016, consulté le 03 novembre 2020 URL : http://journals.openedition.org/asp/4709; DOI : https://doi.org/10.4000/asp.4709

Ce document a été généré automatiquement le 3 novembre 2020.

Tous droits réservés 


\title{
Réflexions sur les termes-valises en anglais de l'économie, de la gestion et de la finance
}

\author{
Reflections on blends in English for economics, management and finance
}

\author{
Catherine Resche
}

Les aspects lexicaux et terminologiques des discours spécialisés dans les domaines de l'économie, de la gestion et de la finance en anglais ont déjà été analysés à de maintes reprises et sous des angles différents (Van der Yeught 2007; Percebois 2010 ; Resche 2013a), et de nouvelles approches, comme la prise en compte des anthroponymes (Resche 2013b), ont été envisagées. Toutefois, il est un aspect de ces domaines qui, s'il a été amplement étudié dans la langue générale, en anglais comme en français, n'a pas encore reçu l'attention qu'il mérite dans l'étude des domaines spécialisés : il s'agit des néologismes à caractère hybride. Or, ces formations que nous appellerons «termesvalises ", et qui résultent d'une imbrication (d'aucuns diront d'un télescopage), sont dignes d'intérêt par l'éclairage qu'elles peuvent nous apporter sur le plan de l'évolution des idées et des pratiques. Dans cette perspective, une veille s'impose pour repérer les nouvelles créations et analyser la signification et la portée de ces amalgames pour ces domaines.

Le corpus qui a nourri cette étude est composé d'un large éventail d'ouvrages de recherche (Morgan 1993 ; Waterman 1993 ; Hock 1995 ; Krugman 1998 ; Levitt \& Dubner 2005; Thomas 2009), d'articles de revues spécialisées (McKinsey Quarterly, Harvard Business Review, Knowledge@Wharton ${ }^{1}$ ) et de manuels d'économie (Samuelson \& Nordhaus 2005; Mankiw 2004²). La cinquantaine d'amalgames lexicaux que nous avons relevés au cours de nos lectures appartiennent tous aux domaines de l'économie, de la gestion et de la finance, domaines dont nous étudions les aspects terminologiques, néologiques et discursifs depuis maintenant trois décennies en anglais de spécialité.

Après avoir fait un bref rappel des questions concernant les diverses dénominations et définitions des amalgames lexicaux, nous présentons le tableau des amalgames retenus et nous les analysons à la lumière du positionnement que nous avons annoncé. Nous 
nous attachons ensuite à faire ressortir la spécificité des " termes-valises » par rapport aux «mots-valises ». Enfin, nous nous intéressons plus particulièrement à quelques exemples qui nous servent à illustrer et à mettre en évidence la nécessité d'accorder une attention plus soutenue à ces formations dans les discours spécialisés, qu'ils soient disciplinaires ou professionnels.

\section{Cadre théorique : questions de dénominations et de définitions}

Les recherches antérieures sur le phénomène de l'amalgamation ou "valisage " (Bonhomme 2009) ont donné lieu à une pluralité de dénominations au fil du temps. La reprise de l'emprunt au français fait par Lewis Carroll au XIX siècle dans Through the Looking Glass avec portmanteau word n'a pas réuni tous les suffrages, même si c'est à partir de cette époque que ce processus lexicogénique a retenu l'attention. Il a, depuis lors, connu un essor marqué et suscité maintes analyses, mais c'est plutôt la dénomination «mots-valises » qui a été préférée parmi une riche liste d'appellations candidates. Certaines des dénominations concurrentes évoquent la métaphore de l'enchâssement, comme «mots-gigognes » ou «mots-tiroirs» (Morier 1998). D'autres mettent en avant le caractère étrange, voire monstrueux (Grésillon 1983), de ces formations considérées comme transgressives sur le plan linguistique: "motscentaures » (Rigaud 1969), «bêtes-à-deux-mots » (Moncelet 1981). D'autres encore, insistent sur leur caractère hybride (ou inhabituel) : c'est le cas de «mots-métis » et "mots téléscopés " ${ }^{3}$ Sans prétendre à l'exhaustivité, nous citerons encore "motssandwich» (Hesbois 1986), «mots articulés » (Galisson 1991) ou «mots composites» (Martin 1976). Enfin, comme il l'explique lui-même, et " pour éviter les termes plus ou moins marqués ", André Clas (1987: 347) a suggéré l'expression "brachygraphie gigogne ».

Ce «trop-plein terminologique» (Sablayrolles 2002) pointe également vers des positionnements divergents quant à la nature et la définition même du concept. MarieFrançoise Mortureux (1995 : 19) présente les mots-valises en termes assez généraux :

on regroupe généralement sous ce terme un ensemble de formations qui consistent à créer un unique lexème à travers diverses manipulations, tenant en général du télescopage, du signifiant de deux lexèmes-bases.

De son côté, Garland Cannon (2000 : 952) propose une définition nuancée, dans laquelle les précautions discursives laissent à penser que la porte est assez largement ouverte :

a process of word formation in which two (or rarely, three) separate source items are telescoped into a new form, which usually exhibits overlapping and retains some of the meaning of at least one of the source items.

Si l'on se fie aux dictionnaires, les définitions sont plus ou moins précises : le Trésor de la langue française se montre très inclusif, avec une définition large du mot-valise qui correspondrait à une "création verbale formée par le télescopage de deux ou (trois mots) existant dans la langue " alors que le Grand Dictionnaire Terminologique du Canada se veut plus restrictif : le mot-valise y est défini comme un «terme simple composé de deux éléments lexicaux réduits, ne conservant que la partie initiale du premier et la partie finale du dernier ». Le site de l'office québécois confirme et précise cette définition : 
Un mot-valise est un mot résultant de la fusion d'éléments empruntés à deux mots. La plupart du temps, ce télescopage à l'origine du mot-valise consiste à combiner la partie initiale d'un mot et la partie finale d'un autre mot, et à unir les sens respectifs de ces deux formes. Le mot-valise, parfois appelé mot-centaure, est donc une sorte de collage formel et sémantique.

Les précisions apportées par les définitions canadiennes vont dans le sens d'une première exigence à caractère morphologique, selon laquelle la troncation doit correspondre à une apocope de la première base-source et/ou une aphérèse de la seconde. Est-ce à dire que des compositions à partir de deux apocopes ou deux aphérèses ne peuvent entrer dans la catégorie des mots-valises? Dans ce cas, certaines des entrées que nous avons pourtant retenues, comme burgernomics ${ }^{4}$ (aphérèse + aphérèse) ou chaord ${ }^{5}$ (apocope + apocope), ne seraient pas recevables. Une deuxième condition, posée par un certain nombre de linguistes, est d'ordre sémantique et porte sur la nécessité d'un lien de coordination entre les deux lexèmes-sources de type « $\mathrm{A}$ est à la fois un B et un C ». Ainsi, si l'on peut dire que stagflation est à la fois stagnation et inflation, cette entrée est envisageable. Enfin, un troisième critère de définition mis en avant, de caractère morpho-phonologique cette fois, fait de l'obligation d'un segment homophone commun une condition sine qua non pour permettre la fusion ou l'interpénétration des deux lexèmes-sources (Fromilhague 1995: 22 ; Beth \& Marpeau $2005: 20$ ). Fabienne Cusin-Berche (1999: note 31) établit d'ailleurs une distinction entre des formations qui répondent à ce critère et pour lesquelles elle réserve la dénomination "mots-valises", et celles qui ne le respectent pas; paradoxalement, pour désigner les compositions imparfaites, elle a recours à un véritable mot-valise (compocation), formé à partir de compo(sition) et de (tron)cation. Julie Makri-Morel (2015) se veut encore plus sélective dans son approche des mots-valises, puisqu'elle insiste sur la nécessité pour l'élément commun de se situer à la jointure des deux bases. $\mathrm{Au}$ regard de ces remarques, slumpflation, dans notre corpus, ne devrait pas être considéré comme mot-valise, puisqu'il ne répond pas au critère d'homophonie partielle permettant la fusion des deux mots-sources.

Toutefois, comme le souligne Vincent Renner (2006: 139), de nombreux chercheurs (Adams, 1973 ; Algeo 1977 ; Clas 1987) semblent s'accorder sur une définition minimale selon laquelle le mot-valise résulte de l'assemblage d'au moins deux lexèmes, dont un perd une partie de son signifiant. Même si d'autres, nous l'avons vu, ressentent le besoin d'ajouter des traits complémentaires, pour V. Renner, l'important est de s'entendre sur un "plus petit dénominateur commun ». C'est d'ailleurs dans cet esprit que nous avons réuni les amalgames que nous allons maintenant présenter.

\section{Présentation et analyse morphologique des mots- valises retenus}

La liste que nous avons retenue comprend une cinquantaine de mots-valises, que nous avons dans un premier temps organisés en fonction des domaines d'application : nous les présentons dans le tableau récapitulatif ci-dessous (tableau 1). Nous avons conscience que la présence de plusieurs termes se terminant en nomics/onomics, est de nature à étonner des chercheurs qui considèrent, comme Bernard Fradin (2015: 55), que les mots-valises et les séries sont antithétiques. Si nous avons laissé de côté Clintonomics, Bushonomics, Obamanomics, etc., qui constituent des exemples de composition cachée ou "fracto-composition" et s'insèrent dans des paradigmes 
constructionnels, nous avons maintenu d'autres entrées composées avec nomics/ onomics, car elles ne signifient pas automatiquement "l'économie selon $\mathrm{X}$ ». Nous reviendrons sur ce point ultérieurement.

Tableau 1 : Entrées prises en compte et classées en fonction des domaines d'application

\begin{tabular}{|l|l|}
\hline Économie & $\begin{array}{l}\text { Agrinomics, Bionomics, Boomernomics, Burgernomics (1986), Crunchonomics, } \\
\text { Duopsony, Ecofinance, Econometrics (Frisch 1930), Econosphere, Econophysics, } \\
\text { Enronomics, Flexinomics, Freakonomics (Levitt \& Dubner 2005), Globaloney } \\
\text { (Krugman 1998), Guesstimate/Guestimate, Ideanomics (Greenspan 2001), } \\
\text { Monopsony, Neuronomics, Oligopsony, Satisficing (Simon 1947), Slumpflation } \\
\text { (Merriam Webster online 1974), Stagflation, Stagility }\end{array}$ \\
\hline $\begin{array}{l}\text { Gestion/ } \\
\text { marketing }\end{array}$ & $\begin{array}{l}\text { Adhocracy (Toffler 1970, puis utilisé par Minzberg), Advertainment, Advertorial, } \\
\text { Agribusiness, Chaord /Chaordic (Dee Hock), Coopetition (Brandenburger \& } \\
\text { flexitime, Frenemies, Glocalization/glocal, Imaginization (Gareth Morgan : the art } \\
\text { of creative management), Infomercial, Infotainment, Nanopreneur, Perkonomics, } \\
\text { Promotainment, Prosumer (Heidi \& Alvin Toffler), Retailtainment (2001 Wal-Mart) }\end{array}$ \\
\hline Finance & $\begin{array}{l}\text { Bancassurance, Bankster, Banktivism, Banktivist, Branker, Creditaholic, } \\
\text { Greenmail, Ledge (fund), Spendaholic, Swaption }\end{array}$ \\
\hline
\end{tabular}

Les caractères gras dans le tableau 1 signalent les termes attestés par une définition dans le domaine spécialisé, créés par des spécialistes du domaine, et qui sont connus, compris et partagés par les acteurs du domaine : nous reviendrons sur ce point dans la partie 3. Nous avons choisi de laisser en caractères maigres les entrées qui ne sont pas réservées aux discours spécialisés puisqu'on peut les retrouver dans la presse semispécialisée (New York Times, The Economist, etc.), d'autant qu'il n'a pas toujours été facile d'identifier les auteurs à l'origine de ces amalgames, même si des exemples comme advertainment, infomercial ou infotainment sont couramment utilisés dans les départements de marketing des entreprises ${ }^{6}$.

Une autre façon d'analyser les mots-valises retenus est de les considérer en fonction de leur formation, indépendamment du sous-domaine auquel ils se rapportent (tableau 2). Nous ne commenterons que les éléments dont la formation nous a posé des problèmes de catégorisation ou qui ont retenu notre attention par une formation moins classique.

Nous nous sommes dans un premier temps interrogée sur la façon d'envisager certaines entrées pour lesquelles l'élément charnière (encore appelé point de bascule) semble être mobile selon l'analyse qu'on en fait. Par exemple, dans Enronomics, faut-il placer la charnière en considérant que le premier élément est intact (Enron/omics) ou considérer qu'il y a apocope et aphérèse (Enr/onomics ${ }^{7}$ ou même Enro/nomics) ? Dans la mesure où le segment identique n'est pas répété, il est difficile de trancher, sauf à se ranger du côté de la définition étroite du mot-valise et à penser qu'il y a ici apocope et aphérèse. Cette remarque vaut également lorsque les deux lexèmes-sources n'ont qu'un seul phonème en commun, comme pour swaption (swap/(op)tion ou swa(p)/(o)ption). Dans le cas de Globaloney, il n'y a aucun doute sur l'apocope pour le premier élément (le premier lexème-source étant globalization), et, même si l'on peut être tenté de penser que le second élément a été conservé dans son intégralité, il semble peu probable qu'il faille 
placer la troncation après la syllabe «glo $»^{8}$. En réalité, le segment commun «bal » n'a pas été répété et sert de charnière, attestant ainsi qu'il y a bien imbrication des deux lexèmes-sources.

Un second motif d'interrogation concerne les trois cas d'emboîtement atypique signalés dans le tableau 2. Si l'on peut considérer que bankster résulte d'une apocope sur bank(er) et d'une aphérèse sur $(g) a n(g) s t e r$, il est également possible d'envisager que la terminaison er étant commune, seules les lettres st sont venues s'imbriquer dans banker pour permettre la compréhension de l'amalgame. Pour l'amalgame branker, de manière analogue, il y a plusieurs segments communs : br(o)ker et banker. En ce qui concerne ledge fund, il y a plus qu'une apocope sur le(verage) et une aphérèse sur (h)edge, puisqu'il faut prendre en compte non seulement les deux lettres finales, mais aussi l'homophonie [d3] portant sur la fin de chacun des mots-sources. À propos d'homophonie, un autre cas à noter est celui de frenemies : dans cet amalgame, il est peut-être tentant, mais erroné, de penser que le deuxième élément de l'amalgame (enemies) est resté intact ; en effet, si le $i$ de la première partie de friend a disparu, on peut considérer que le segment commun aux deux lexèmes-sources est en, et, là encore, il s'agit d'un cas d'homophonie. 
Tableau 2 : Récapitulatif des formations des amalgames pris en compte

\begin{tabular}{|c|c|}
\hline Apocope + aphérèse & 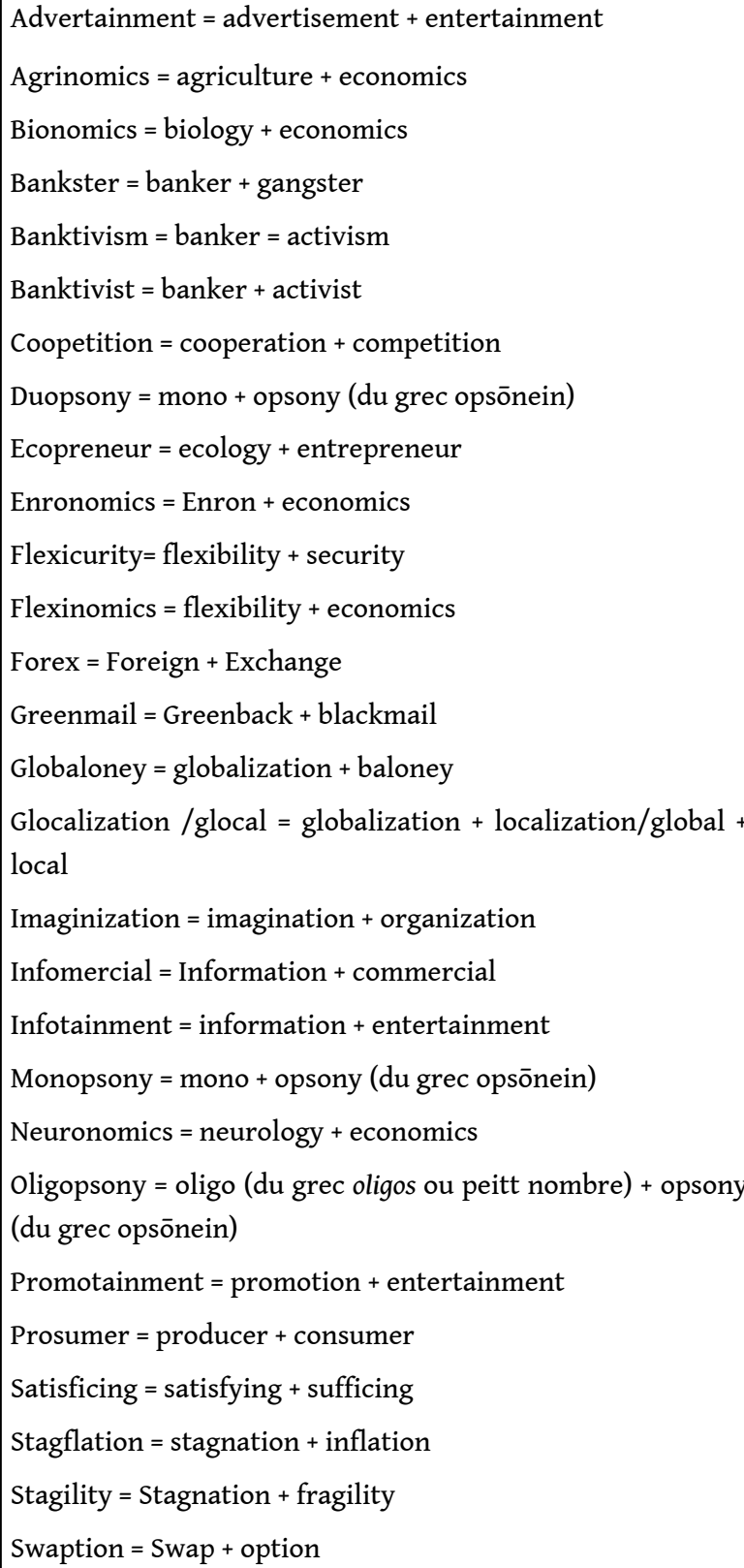 \\
\hline $\begin{array}{l}1^{\text {er }} \text { élément intégral + apocope sur le } \\
\text { second élément sans segment } \\
\text { commun }\end{array}$ & $\begin{array}{l}\text { Crunchonomics, Freakonomics, Ideanomics, Nanopreneur } \\
\text { Perkonomics, Retailtainment, Slumpflation }\end{array}$ \\
\hline Apocope + second élément intégral & $\begin{array}{l}\text { Agribusiness, Bancassurance }{ }^{9} \text {, Ecofinance, Ecomanagement } \\
\text { Econosphere, Econophysics, Flex(i)time }\end{array}$ \\
\hline Aphérèse + aphérèse & Burgernomics, Boomernomics \\
\hline Apocope + apocope & Chaord, Forex \\
\hline
\end{tabular}




\begin{tabular}{|l|l|}
\hline Formations plus rares & $\begin{array}{l}\text { Au niveau de l'emboitement : Bankster (Banker + gangster), } \\
\text { Branker, Ledge (fund) = leverage + hedge (fund) } \\
\text { Au niveau du segment commun fondé sur l'homophonie, et qui } \\
\text { affecte l'orthographe d'un des éléments : frenemies (friends + } \\
\text { enemies) } \\
\text { Ajout de suffixe : Chaordic, Ecopreneurship } \\
\text { Ajout de voyelle épenthétique : Creditaholic, Spendaholic } \\
3 \text { composants : Adhocracy (premier élément intégral composé } \\
\text { de deux mots + aphérèse. Ad hoc + bureaucracy); } \\
\text { Composants latins et grecs : adhocracy, duopsony, monopsony, } \\
\text { oligopsony }\end{array}$ \\
\hline
\end{tabular}

Une troisième difficulté illustre la nécessité de vérifier les bases-sources afin d'éviter de tomber dans des pièges : Greenmail, par exemple, peut-être trompeur si l'on considère green comme un élément intégral ; en réalité, il y a bien apocope sur le premier élément qui est à l'origine greenback et une aphérèse sur le second, blackmail. La définition fournie par le dictionnaire Merriam Webster en ligne ${ }^{10}$ est claire à ce sujet : "the practice of buying enough of a company's stock to threaten a hostile takeover and reselling it to the company at a price above market value; also: the money paid for such stock. "

Une quatrième question concerne les fracto-morphèmes que Jean Tournier (1985: 86) définit comme « un fragment de lexie qui la représente dans un mot construit ». Les fractomorphèmes nomics ou onomics (tableau 3) sont assez nombreux dans le corpus et servent de matrice à des créations lexicales: si nous n'avons pas retenu ceux qui évoquent systématiquement les choix de politique économique d'un personnage public (les plus récents sont Abenomics pour le Japon et Renzinomics pour l'Italie), nous avons maintenu Enronomics, pour lequel les choses se passent quelque peu différemment, dans la mesure où Enron évoque bien plus que l'entreprise elle-même: Enronomics fait référence à des pratiques peu recommandables de créativité en matière de comptabilité ("creative accounting») et cet amalgame a donc un sens assez précis pour les spécialistes. De même, les autres occurrences conservées dans le tableau dénotent bien autre chose, qu'il importe de définir, ce que nous ferons en abordant les aspects sémantiques dans la partie 3.

Tableau 3. Amalgames terminés en nomics / onomics

\begin{tabular}{|l|l|l|}
\hline $\begin{array}{l}\text { Nom propre + nomics, mais avec un sens différent de } \\
\text { «'économie selon X... » }\end{array}$ & $\begin{array}{l}\text { Risque de faux sens ou } \\
\text { contresens }\end{array}$ & Autres \\
\hline Enronomics & $\begin{array}{l}\text { Agrinomics } \\
\text { Boomernomics } \\
\text { Flexinomics } \\
\text { Freakonomics } \\
\text { Perkonomics }\end{array}$ & $\begin{array}{l}\text { Bionomics } \\
\text { Burgernomics } \\
\text { Crunchonomics } \\
\text { Ideanomics } \\
\text { Neuronomics }\end{array}$ \\
\hline
\end{tabular}

Enfin, il convient de noter des formations plus atypiques à partir de composants d'origine grecque ou latine. Ainsi, adhocracy ${ }^{11}$, qui s'oppose à bureaucracy, est composé 
d'une expression empruntée au latin ad hoc et d'un fractomorphème emprunté au grec

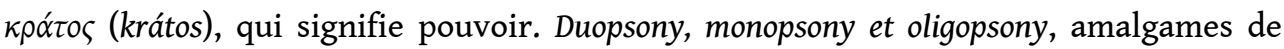

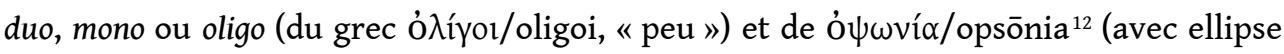
d'une des voyelles communes o à la charnière), illustrent aussi la possibilité pour ces termes-valises de servir de base à la formation d'autres termes de la même famille par ajout de suffixe : duopsonistic, etc.

On notera aussi, au niveau des mots-valises servant de support à des suffixes, les cas de ecopreneur/ecopreneurship, nanopreneur/nanopreneurship, ecomanager/ ecomanagement ainsi que les paires adjectif/nom constituées par glocal/glocalization ou encore nom/nom pour banktivism/banktivist.

Après ces remarques sur les traits morphologiques des entrées analysées, il convient d'envisager les aspects sémantiques et de nous intéresser plus particulièrement aux termes-valises.

\section{La spécificité des «termes-valises »: considérations d'ordre sémantique et changements théoriques}

Le mot-valise, de manière générale, attire l'attention par son caractère incongru. En effet, comme nous l'avons vu, il ne correspond pas aux formations classiques de mots : à la différence du mot composé qui procède par juxtaposition, le mot-valise est formé par imbrication. Alain Finkielkraut (1979) voit dans le mot-valise une source de créativité à laquelle tout un chacun peut accéder et, pour lui, le pêle-mêle et la confusion engendrés ont une saveur. Le recours à ce type de néologismes est d'ailleurs assez fréquent en littérature (Lewis Carroll, mais également Rabelais, Rostand, Rimbaud, Etiemble, Montherlant, et Céline, par exemple) ${ }^{13}$, où les auteurs se plaisent à jouer sur la transgression linguistique et à explorer les possibilités offertes par la langue ; cette figure de style, qui invite le lecteur à un certain décodage, puisqu'elle condense plusieurs significations en un seul mot, est également mise à profit dans la publicité ou la presse. Le but est alors de marquer les esprits en créant un effet particulier, de nature comique, ironique, ou de la frapper par son étrangeté.

De manière générale, le caractère surprenant, voire dérangeant ou déstabilisant $d u$ mot-valise invite à reconsidérer les habitudes, à faire bouger les lignes, à penser différemment. Pour reprendre l'expression de Marc Bonhomme, les mots-valises sont des " passeurs de frontières ». Ils peuvent d'ailleurs tellement bien passer les frontières que certains finissent par être lexicalisés et trouvent leur place dans les dictionnaires de langue générale, de sorte que nous avons cessé de les percevoir comme des motsvalises ou des figures de style : c'est le cas de « courriel » ou encore de " gélule ».

En réalité, le mot-valise signifie plus que la somme de ses constituants : en dehors des aspects ludiques qui peuvent lui être attachés (Renner 2006; Vorger 2015), il semble créer un véritable espace d'interface. Il est souvent créé de manière à remplir un vide lexicologique, dans une démarche consciente et volontaire. Quand son apparition peut être datée et son auteur identifié, il est possible de le replacer dans son contexte historique et social. Il convient alors d'en analyser la valeur pragmatique, énonciative et sociolinguistique. 
Dans les domaines de spécialité, le mot-valise peut devenir «terme-valise » s'il dénomme un nouveau concept, renvoie à un phénomène ou une pratique particulière, ouvre, en quelque sorte, de nouveaux horizons. Le "terme-valise", résultat d'une démarche onomasiologique volontaire, puisque son auteur part de la notion nouvelle pour trouver une façon de la dénommer, s'apparente alors à ce que Guy Rondeau (1984) appelle néonymes. Le télescopage qui lui a donné naissance crée de nouveaux espaces conceptuels, et donne lieu à une restructuration des représentations; le fait de créer un terme-valise, de repenser la théorie, d'exprimer ainsi une autre manière de voir les choses, permet de nourrir le débat et d'avancer. Dans certains cas, le terme-valise peut être considéré comme révolutionnaire puisqu'il peut refléter un tournant dans la théorie, une remise en question du statu quo pouvant donner lieu à un véritable changement de paradigme au sens kuhnien du terme ; plus souvent, il annonce et ouvre de nouvelles perspectives de recherche.

Considérons, par exemple, satisficing, terme-valise résultant de l'amalgame entre satisfy et suffice qui s'inscrit dans le cadre des études sur la prise de décision d'un agent économique et que nous devons à l'économiste Herbert Simon $(1947,1957)$, lauréat du Prix de la Banque de Suède pour l'économie en $1978^{14}$. Alors que, pendant longtemps, on avait pensé que l'homo oeconomicus était rationnel et capable de prendre des décisions qui lui permettaient d'optimiser ses choix, H. Simon a voulu tenir compte des limites en termes d'information ou de temps qui empêchent de faire un choix optimal et il a ainsi substitué la notion de solution satisfaisante à celle de solution optimale. Pour exprimer ce changement d'approche, et servir la théorie de la rationalité limitée (bounded rationality), il a eu recours à «satisficing ». Dans son discours lors de réception du Prix de la Banque de Suède, il revient sur cette notion (Simon 1979: 498) : «[...] decision makers can satisfice either by finding optimum solutions for a simplified world, or by finding satisfactory solutions for a more realistic world."

Imaginization (Morgan 1993), autre exemple, montre une nouvelle voie, celle du management créatif, qui invite à repenser tous les aspects du management, de la structure de l'organisation à la stratégie, en passant par le travail d'équipe, les produits et les services. Il suffit de reconsidérer les métaphores sous-jacentes, d'envisager de nouvelles « images » en utilisant une ressource sous-employée, celle du lobe droit du cerveau, de l'imagination, pour ne pas se laisser emprisonner par les règles, habitudes et modes de pensée traditionnels. Dans le même ordre d'idée, le néologisme ideanomics que nous devons à Alan Greenspan traduit bien l'idée d'une transformation radicale de l'économie et le passage d'une économie fondée sur le capital physique à une économie fondée sur le capital intellectuel. Aucun autre terme existant n'était satisfaisant à ses yeux pour expliquer au Congrès américain que, désormais, à l'échelle planétaire, nous étions entrés dans une nouvelle ère où l'économie était dominée à tous les niveaux par les idées, l'innovation, la créativité, le capital intellectuel, le savoir, la propriété intellectuelle; il convenait donc de combler le vide terminologique constaté.

Certains «termes-valises" traduisent l'évolution de la discipline en pointant l'interdisciplinarité croissante à l'œuvre dans les domaines scientifiques; c'est le cas des amalgames ecofinance ${ }^{15}$, neuronomics, econophysics ou bionomics qui dénomment de nouvelles branches de la recherche à la croisée des chemins entre champs disciplinaires. Ils traduisent l'interface entre deux ou plusieurs domaines (Humbley 2006) et ouvrent de nouvelles perspectives de recherche. Le terme-valise peut aussi souligner la pénétration de nouvelles tendances au sein d'un domaine de spécialité 
comme la gestion : c'est ainsi que la théorie du chaos, qui a trouvé des échos dans de nombreuses disciplines, a ouvert de nouvelles perspectives en matière de gestion des entreprises par le biais du terme-valise chaord et de l'adjectif chaordic. Dee Hock ${ }^{16}$, auteur de ce «terme-valise », explique ainsi le concept, qui ne correspond ni à l'ordre rigide, ni au chaos, mais relève de modes de pensée inspirés de la biologie et de la physique (1995: 1) :

By Chaord, I mean any self-organizing, adaptive, non-linear, complex system, whether physical, biological, or social, the behaviour of which exhibits characteristics of both order and chaos or, loosely translated to business terminology, cooperation and competition.

Certains amalgames sont particulièrement intéressants en ce qu'ils se font le reflet d'une société et d'un monde en évolution. Ainsi, glocalization (glo(balization) + (lo)calization) est particulièrement marqué par le contexte dans lequel opèrent désormais les entreprises. Il fallait trouver un moyen d'exprimer de manière forte la nécessité pour les entreprises de penser à l'échelle de la planète, tout en s'adaptant aux marchés locaux et aux attentes particulières de leurs clients potentiels. Ce terme-valise incarne bien le défi qui consiste à ne privilégier aucun des deux pôles représentés par les deux constituants d'origine, et à réconcilier ce qui semble opposé au départ. Inspiré par le même contexte planétaire, globaloney traduit une autre fonction des termesvalises : leur vocation à susciter ou nourrir le débat. Bien que créé par Clare Booth Luce ${ }^{17}$ pour dénoncer des propos peu clairs sur la géopolitique, il a été repris pour l'économie par Paul Krugman (1998) pour dénoncer le fait que la mondialisation est trop souvent mise en avant pour expliquer tous les problèmes.

Si le sens d'un mot-valise est le plus souvent transparent, il n'en est pas de même des termes-valises qui prêtent souvent à confusion et doivent être replacés dans leur contexte pour que toute ambiguïté soit levée. Il faut, comme pour un terme classique, en vérifier la définition. Ainsi, flexinomics est trompeur si on pense à flextime $e^{18}$, autre terme-valise, ou encore flexicurity. Le lecteur retrouvera aisément le composant flexible, mais il lui faudra s'assurer du sens exact de l'amalgame : « A practice of renting or leasing so as to remain financially flexible and nimble in bad economic times ». Il s'agit donc d'une offre d'options de financement pour faciliter l'acquisition de biens que certains acquéreurs potentiels ne pourraient payer comptant. Toute une série de mesures sont alors prises pour répondre à une période de crise, et le terme-valise qui rend le mieux compte de ces nouvelles dispositions pour s'adapter à la crise est crunchonomics. De même, perkonomics est faussement transparent car rien ne précise à qui s'adressent les avantages en question. On pourrait assurément penser qu'il s'agit d'une technique pour récompenser les bons employés, alors qu'il s'agit de repenser la relation de l'entreprise avec ses clients pour les fidéliser ${ }^{19}$. Boomernomics ${ }^{20}$ peut également poser des problèmes d'interprétation. Toujours à propos des amalgames contenant le fracto-morphème onomics, Freakonomics (Levitt \& Dubner 2005), titre d'un ouvrage co-écrit par un économiste de l'Université de Chicago et un journaliste du New York Times Magazine, n'a évidemment rien de monstrueux, mais, en adéquation avec le sens donné à freak dans le contexte de la contre-culture des années 1960 aux États-Unis, cet ouvrage offre une vision peu traditionnelle de l'économie, en explorant la face cachée des choses de la vie courante.

Si l'on s'intéresse à ecopreneur, par référence à entrepreneur, on peut sans doute comprendre qu'il s'agit de quelqu'un qui crée une entreprise dont les produits ou services sont en relation avec le respect de la nature, mais que dire de "nanopreneur ", 
qui laisserait logiquement penser qu'il s'agit d'un créateur d'entreprise dans le secteur des nanotechnologies, alors qu'en réalité, il fait référence à un créateur de très petite entreprise (TPE). Le terme-valise « econosphere ${ }^{21} »$, créé par l'économiste Craig Thomas (2010:1), n'est pas non plus vraiment transparent.

Il est un autre aspect des termes-valises qu'il faut prendre en compte : la possibilité que plusieurs interprétations soient en concurrence, ou que leur sens évolue au fil du temps, d'où la nécessité de vérifier leur acception en contexte, y compris sur l'axe diachronique. Ainsi, satisficing, que nous avons déjà évoqué, est tantôt utilisé avec son sens premier formé à partir de satis(fy) + (sufffice, tantôt interprété comme s'il résultait de l'amalgame satis(fy) + (sacri)fice. Son auteur, Herbert Simon, avait pourtant été clair sur l'idée de «good enough»:

Whereas economic man maximizes - selects the best alternative from among all those available to him, his cousin, administrative man, satisfices - looks for a course of action that is satisfactory or "good enough". (1997 [1947] : 29)

La notion est actuellement exposée différemment aux étudiants d'un programme de $\mathrm{MBA}^{22}$ :

Satisficing can be viewed as a construct of the words satisfy and sacrifice. This way, the notion of loss is introduced into the calculation. Rather than viewing the decision point from the perspective of how little effort will suffice, this interpretation shifts the focus to one of how much you are willing to sacrifice by making decisions a certain way.

Le cas de prosumer est également assez révélateur : créé dans les années 1980 par Alvin Toffler pour faire référence à un consommateur référent qui est partie prenante dans l'élaboration d'un produit (en donnant son avis, en suggérant des façons de " customiser " le produit), il correspondait au départ à l'amalgame des deux lexèmessources pro(ducer) et (con)sumer. Toutefois, certains, dans le cadre du Web 2.0, le comprennent différemment, comme l'amalgame de pro(fessional) + (con)sumer, considérant que les utilisateurs actifs ajoutent du contenu et accèdent au statut de professionnels.

Les termes-valises, nous l'avons vu, peuvent servir d'indices quant à des tournants importants dans la théorie. Ils sont également intéressants lorsqu'ils permettent de repérer une évolution ou un changement radical dans les pratiques.

\section{Termes-valises et changements dans les pratiques}

À mi-chemin entre théorie et pratiques, le concept de coopetition ${ }^{23}$ nous offre l'occasion de souligner l'impact de cette catégorie d'amalgames qui résultent d'un rapprochement inattendu de deux concepts apparemment incompatibles, ici la coopération et la concurrence (competition). Le nouveau concept équivaut donc à une véritable révolution cognitive. Mener des recherches sur la coopétition conduit forcément à un réexamen de l'existant et à une nouvelle définition des normes d'interaction entre les entreprises. Pour Adam M. Brandenburger et Barry J. Nalebuff, auteurs de l'ouvrage éponyme (1995), la clé est de considérer les affaires comme un jeu, ce qui permet d'analyser les aspects relevant de la concurrence et ceux qui peuvent être mis à profit dans une coopération éventuelle. Le but est de se faire une idée plus précise de la situation dans laquelle l'entreprise évolue. En empruntant quelques concepts de base à la théorie des jeux, on peut se donner de meilleures chances de tirer son épingle du jeu. Le termevalise coopetition va de pair avec deux autres termes-valises, frenemies et froes, eux aussi 
composés de mots-sources ordinairement irréconciliables et qui sont communément utilisés dans le domaine de la stratégie d'entreprise.

Dans le domaine du marketing, un certain nombre de termes-valises sont apparus, avec des allers et retours entre discours des médias et discours des entreprises. Comme le montre le tableau 4 , ces termes attestent du décloisonnement des genres, ce qui peut traduire un changement dans les attentes et dans les habitudes du public cible. Il est effectivement difficile désormais de concevoir une publicité qui ne soit pas divertissante ou ne contienne pas une part d'information pour répondre aux besoins des consommateurs plus avertis. Les amalgames présentés dans le tableau 4 illustrent bien ces nouveaux genres hybrides qui ont été imaginés pour accompagner l'évolution de la société et s'adapter aux nouveaux consommateurs mieux informés, plus exigeants, et en intégrant les possibilités offertes par les nouvelles technologies du multimédia. D’autres amalgames sont ainsi nés, comme webzines, fanzines et webisodes qui signalent l'émergence de nouveaux genres de discours au niveau des entreprises et ouvrent de nouvelles perspectives et de nouveaux espaces de réflexion en matière de gestion des clients.

Tableau 4. Amalgames dans le domaine du marketing

\begin{tabular}{|l|l|}
\hline advertainment & infotainment \\
\hline advertorial & promotainment \\
\hline infomercial & retailtainment \\
\hline
\end{tabular}

Enfin, dans le domaine de la banque et de la finance, on note un certain nombre de termes-valises qui, là encore, accompagnent événements ou changements et servent d'indices ou de témoins pour l'observateur. Ainsi, depuis les années 1930 et la Grande Dépression, le mot-valise banksters a une connotation négative et renvoie aux acteurs peu scrupuleux des institutions financières, qui sont alors assimilés à des gangsters. Abondamment repris par la presse britannique à l'occasion de la crise de 2008, il atteste de la piètre réputation des acteurs de la banque et de la finance dans le grand public. Il $\mathrm{y}$ a fort à parier que le nombre de ses occurrences pourrait servir de baromètre des scandales financiers, en signalant l'irresponsabilité des montages financiers et le manque de sens de l'éthique des principaux acteurs.

Toutefois, les termes-valises formés en partie à l'aide de banker n'ont pas automatiquement une connotation négative. Branker, par exemple, bien que moins transparent pour le profane, est totalement neutre et dénote simplement un décloisonnement du métier de courtier (broker) et du métier de banquier en matière d'offre d'investissement dans des fonds hypothécaires. De tels néologismes remplissent donc un vide terminologique pour évoquer la diversification des attributs des banques par rapport à ce qui était considéré jusqu'alors comme leur cœur de métier.

Deux autres amalgames, très récents, ont retenu notre attention. Il s'agit de banktivism et banktivist qui peuvent, au premier regard, induire en erreur: en effet, si l'on comprend d'emblée l'amalgame entre banque et activisme, on peut être tenté de penser, par analogie avec les termes activist funds ou shareholder activists ${ }^{24}$, à des banques particulièrement avides de gains. On est alors bien loin du sens de ce terme-valise. On 
pourrait aussi penser aux petits groupes d'activistes qui dénoncent les pratiques peu transparentes des banques et, munis de chiffons, seaux et balais, et déguisés en gentils clowns au nez rouge organisent des opérations non violentes dans certaines succursales pour « faire le ménage et nettoyer l'argent sale » de manière symbolique. Là encore, le véritable sens du terme-valise est bien éloigné de cette interprétation. L'activisme dont il est question ici n'a pas pour objet le profit à tout prix ; l'initiative ne vient pas non plus de clients mécontents ou du public, mais de certains banquiers et acteurs de la finance eux-mêmes, comme en attestent les explications ci-dessous :

Banktivism reflects a specific form of activism. Since the passing of the Community Reinvestment Act, and through the recent 2008 recession, some banks and bankers have chosen to use their influence among their stakeholders to make a difference in the communities they serve by supporting activities aimed at promoting and directing social, political, economic, or environmental change.

Banktivists are banks and bankers that participate in activities that support their local communities, or the greater community at large, in an effort to produce benefit for local residents, small businesses, and the nonprofit community.

Banktivists are bankers at all levels [...]: bank directors, CEOs, branch personnel, and support staff. Banktivists are concerned with maintaining an educated, healthy, vibrant, and bankable community of consumers, small businesses, and nonprofits [...], ensuring that the needs of the community are met while earning a fair return on investment. Banktivists are concerned with issues important to the community, including but not limited to job creation, living wages, social and economic justice, education, health, and overall community development. Banktivists are concerned with treating the community in a respectful and dignified manner. ${ }^{25}$

La création des amalgames banktivist et banktivism doit être replacée dans le contexte dans lequel elle est intervenue, à savoir un contexte de crise de confiance dans les banques de la part du public, qui reproche à l'économie de ne plus remplir sa fonction première : être au service des hommes. Le monde des banques et de la finance est, aux yeux de beaucoup de citoyens, régi par l'appât du gain et la notion d'éthique financière semble devenue un oxymore. C'est précisément pour tenter de démontrer le contraire que les banktivists se sont mobilisés. Ce mouvement encourage le développement d'une offre bancaire solidaire, d'une finance éthique et responsable, capable de soutenir des activités locales, par exemple par le biais de la micro-finance, et véritablement désireux de se mettre au service des hommes plutôt que d'aider au profit de quelques-uns. L'avenir dira si ce courant aura simplement été un sursaut de conscience morale de la part de ses adeptes ou s'il sera de nature à réintroduire durablement l'éthique dans la finance.

\section{Conclusion}

Cette étude a eu pour objet de souligner l'intérêt que les amalgames présentent dans les domaines et discours de spécialité où ils ne sont pas encore suffisamment pris en compte par les chercheurs anglicistes. S'ils ne sont pas tous transparents dans leur composition ou dans leur signification pour le néophyte, une analyse in situ permet de lever les ambiguïtés, comme en attestent les citations à propos de satisficing. Il ne s'agit pas seulement de les considérer dans leur contexte textuel, mais également de prendre en compte ces unités dans leur contexte historique et culturel, et dans leur dimension pragmatique et sociolinguistique. Ces formes, créées à un moment donné pour répondre à des besoins de communication, combler un vide terminologique, repenser la théorie ou les pratiques en ouvrant de nouveaux espaces de réflexion, sont nombreuses 
à pouvoir être considérées comme des termes. Au-delà des effets de mode, il convient donc d'appréhender ces amalgames comme des témoins ou moteurs potentiels de l'évolution des connaissances dans un champ donné du savoir, et un moyen de prendre le pouls de nouvelles attentes de la société. En repoussant les frontières, en bousculant les idées reçues, en réconciliant des concepts apparemment opposés, les amalgames libèrent l'imagination, forcent à penser autrement et contribuent à faire avancer théories et pratiques comme l'ont résumé Albert Einstein et Léopold Infeld (1966 [1938] : 92) : "To raise new questions, new possibilities, to regard old problems from a new angle, requires creative imagination and marks real advance in science ».

Nous espérons avoir convaincu le lecteur que les termes-valises, en tant que marqueurs potentiels de l'évolution des idées, des changements intervenus à une certaine période dans la société, dans le monde des banques, des entreprises ou dans la théorie économique, méritent toute notre attention en ce qu'ils jouent un rôle d'interface. Cette étude n'a pas pu prendre en compte une perspective contrastive, mais il serait sans doute intéressant que les spécialistes de linguistique française et d'autres langues se penchent sur la question de l'adaptation ou de l'emprunt de ces formes par les professionnels non anglophones dans leur discours. La question est aussi de savoir si ce phénomène est présent dans tous les domaines, et dans les mêmes proportions, ou s'il reste limité à quelques sphères spécialisées. Quoi qu'il en soit, il serait dommage d'ignorer les termes-valises dans l'étude des discours spécialisés, et il semble utile de les inclure à toute entreprise de veille terminologique.

\section{BIBLIOGRAPHIE}

ADAMS, Valérie. 1973. An Introduction to Modern English Word Formation. Harlow : Longman.

ALGEO, John. 1977. « Blends, a structural and systemic view ». American Speech 52/1, 47-64.

BETH, Axelle \& Elsa MARPEAU. 2005. Figures de style. Paris : Librio.

BONHOMME, Marc. 2009. « Mot-valise et remodelage des frontières lexicales ». Cahiers de praxématique, consulté le 11 août 2014. <http://praxematique.revues.org/1091>.

Brandenburger, Adam M. \& Barry J. NALeBufF. 1996. Co-opetition. New York : Currency/Doubleday.

CANNon, Garland. 2000. « Blending ». In Booij, G. E., C. Lehmann \& J. Mugdan (dir.), Morphology: An International Handbook on Inflection and Word-Formation, Vol. 1. Berlin/New York : Walter de Gruyter, 952-956.

CLAS, André. 1987. « Une matrice lexicologique universelle : la brachygraphie gigogne ». Meta : le journal des traducteurs/Meta: Translators Journal 2/3, 347-355.

CUSIN-BERCHE, Fabienne. 1999. «La notion d'unité lexicale en linguistique et son usage en lexicologie ». Linx 40, consulté le 31 octobre 2014. <http://linx.revues.org/730>.

EINSTEIN, Albert \& Léopold INFELD. 1966 [1938]. The Evolution of Physics. New York : Simon \& Schuster.

FINKIELKRAUT, Alain. 1979. Ralentir! Mots-valises ! Paris : Seuil. 
FRADIN, Bernard. 2015. « Les mots-valises : jeux et enjeux ». Neologica 9, 35-60.

FROMILHAGUE, Catherine. 1995. Les Figures de style. Paris : Nathan.

GALISSON, Robert. 1991. De la langue à la culture par les mots. Paris : Clé international.

GALISSON, Robert. 2000. «La pragmatique lexiculturelle pour accéder autrement à une autre langue par un autre lexique ». Mélanges CRAPEL 25, 47-73.

GRÉSILLON, Almuth. 1983. « Mi-fugue, mi-raison. Dévaliser les mots-valises ». Communiversation, DRVAV 29, 83-107.

HESBOIS, Laure. 1986. Les Jeux de langage. Ottawa : Éditions de l'université d'Ottawa.

носк, Dee. 1995. « The Chaordic Organization: Out of Control and Into Order ». World Business Academy Perspectives 9/1, 5-18.

HUMBLEY, John. 2006. « La néologie : interface entre ancien et nouveau ». In Greenstein, R. (dir.), Langues et cultures : une histoire d'interface. Paris : Publications de la Sorbonne, 92-103.

Krugman, Paul, 1998. The Accidental Theorist: And Other Dispatches From the Dismal Science. New York : W.W. Norton \& Co.

LeVITT, Steven D. \& Stephen J. DUBNER. 2005. Freakonomics. Londres : Penguin Books.

MAKRI-MOREL, Julie. 2015. « Mots-valises : quand les segments communs se font la malle... ». Neologica 9, 61-79.

MANKIW, N. Gregory 2004. Principles of Economics. 3rd edition, Farmington Hills, MI : Thomson Southwestern.

MARTIN, Jean-Paul. 1976. « La condensation ». Poétique 26, 180-206.

MONCELET, Christian. 1981. Mots-valistes : expédictionnaire illustré, drôlatique, poétique, satirique et grave de bêtes-à-deux-mots. La Roche Blanche : Éditions Bof.

MORGAN, Gareth. 1993. Imaginization: the Art of Creative Management. Newbury Park, CA : Sage.

MORIER, Henri. 1998. Dictionnaire de poétique et de rhétorique. Paris : Presses universitaires de France, coll. « Grands Dictionnaires ».

MORTUREUX, Marie-Françoise. 1995. «Les vocabulaires scientifiques et techniques ». Les Cahiers du Cediscor 3,13-26.

PERCEBOIS, Jacqueline. 2010. «Étude diachronique de termes clés de l'économie du développement en anglais et en français : entre réalisme et euphémisation ». In Jamet, D. \& M. Jobert (dir.), Empreintes de l'euphémisme, Tours et détours. Paris : L'Harmattan, 181-205.

RENNER, Vincent. 2006. « Dépasser les désaccords : pour une approche prototypiste du concept d'amalgame lexical. Le désaccord ». Publications de l'AMAES, 137-147, Consulté le 20 octobre 2014. $<$ halshs- 00512302>.

RESCHE, Catherine. 2013a. Economic Terms and Beyond: Capitalising on the Wealth of Notions. Collection Linguistic Insights $n^{\circ} 176$. Berne : Peter Lang.

RESCHE, Catherine 2013b. « Les anthroponymes en économie : une autre voie d'accès à un domaine de spécialité et à sa culture ». Neologica 7, 137-155.

RIGAUD, André, 1969. « Les mots-centaures ». Vie et Langage 202, 54-57. 
RONDEAU, Guy. 1984 [1981]. Introduction à la terminologie. $2^{\mathrm{e}}$ édition, Chicoutimi : Gaëtan Morin Éditeur.

SABLAYROLLES, Jean-François. 2002. «Des néologismes au signifiant très significatif ». In Gruaz, C. (dir.), Quand le mot fait signe. Rouen : Dyalang, Université de Rouen, 97-117.

SAMUElson, Paul A. \& William D. NoRDHAus. 2005. Economics, 18th edition. New York : McGraw Hill. SIMON, Herbert. 1957. « A Behavioral Model of Rational Choice ». Models of Man, Social and Rational: Mathematical Essays on Rational Human Behavior in a Social Setting. New York : Wiley.

SIMON, Herbert. 1979. «Rational decision making in business organizations ». American Economic Review 69, 493-513.

SIMON, Herbert. 1997 [1947]. Administrative Behavior: A Study of Decision-Making Processes in Administrative Organization. $4^{\mathrm{e}}$ éd. New York : The Free Press/Simon \& Schuster.

THOMAs, Craig. 2009. The Econosphere. Upper Saddle River, NJ : FTPress/Prentice Hall.

TOFFLER, Alvin. 1970. Future Shock. New York : Random House.

TOURNIER, Jean. 1985. Introduction descriptive à la lexicogénétique de l'anglais contemporain. Paris, Genève : Champion-Slatkine.

VAN der YeUGHT, Michel. 2007. «The metaphors of financial bubbles in British and American discourses ». ESP Across Cultures 4, 66-79.

VORGER, Camille. 2015. « Anthropole, Unithèque, Nespresso et autres mots composites dans l'espace universitaire et urbain de Lausanne ». Neologica 9, 133-152.

WATERMAN, Robert H. 1993. Adhocracy. New York : W.W. Norton \& Company, Inc.

\section{NOTES}

1. Knowledge@Wharton est la revue spécialisée en ligne de la Wharton School of Business (Université de Pennsylvanie) ; cette revue s'intéresse aux questions liées à la gestion des entreprises et au management.

2. Nous avons eu accès aux réactualisations en ligne des ouvrages.

3. Ces deux dénominations émanent du Canada http://www.btb.termiumplus.gc.ca/.

4. (Ham)burger + (eco)nomics

5. Cha(os) + ord(er)

6. Il est d'ailleurs évident qu'ils soulignent un changement radical dans la conception des relations avec les clients: les frontières entre information et publicité ludique ont indéniablement bougé pour tenir compte des attentes du public et de l'évolution de la société.

7. Nous convenons que cette option est difficilement envisageable, pour des raisons de morphophonologie.

8. D'ailleurs, l'accent principal de globaloney est bien sur cette première syllabe comme dans global.

9. On notera l'emprunt tel quel au français, la France ayant été à l'origine de ce système.

10. <http://www.merriam-webster.com/dictionary/greenmail> (Consulté le 14 novembre 2014).

11. Créé par Alvin Toffler (1970 : 144), ce terme décrit un mode d'organisation particulier : «the fast-moving, information-rich, kinetic organization of the future, filled with transient cells and extremely mobile individuals ". Ce type de gestion fait appel à l'initiative individuelle et à l'auto-organisation, par opposition aux règles strictes et à la hiérarchie qui caractérisent la bureaucratie. Plus tard, 
Robert H. Waterman, Jr. définit le concept sur la couverture de son ouvrage éponyme par ces mots : " any form of organization that cuts across normal bureaucratic lines to capture opportunities, solve problems, and get results. »

12. Qui signifie achat de nourriture.

13. Cités par Gallisson (2000: 54 ).

14. On ne parle pas de prix Nobel en économie car Alfred Nobel n'avait pas inclus l'économie dans la liste des disciplines à récompenser.

15. Il s'agit ici de voir comment les recherches sur les écosystèmes peuvent être appliquées à la finance pour déployer des stratégies de résilience et, inversement, comment les outils propres à la finance mondiale peuvent avoir une utilité pour la gestion des écosystèmes.

16. Fondateur et premier PDG de Visa

17. élue en 1942 au Congrès américain (House of Representatives) pour le Connecticut, puis ambassadrice au Brésil.

18. Ou sa variante flexitime. Ces deux termes font référence à la possibilité donnée aux employés d'organiser leur journée de travail en fonction de leurs contraintes familiales (commencer plus tard et finir plus tard, par exemple, ou vice-versa).

19. A marketing technique that involves adding new perks and privileges to a brand's regular offerings, in an attempt to satisfy a customer's need for "novel forms of status and/or convenience."

20. Il ne s'agit pas de la façon dont les baby-boomers gèrent leur argent, mais d'une stratégie d'investissement qui cible les produits ou services dont les baby-boomers auront besoin en vieillissant : When they were born, boomers were the largest generation in history. Boomernomics assumes that as this generation ages, demand will increase for geriatric medical products as well as for luxury products like vacation homes. Boomernomics is a long-term investment strategy because it assumes that demand (and therefore profits) for these products will increase only gradually over time. Farlex Financial Dictionary, 2012

21. Selon Craig Thomas, "the Econosphere is our social environment, where we work, live, raise our families, and govern ourselves. We need to start thinking about the economy as a holistic, natural system. To those who are inclined to see it, it is breathtaking choreography on a global scale with billions of performers, each one in character, playing his or her unique role so that the entire ensemble shines". <http://changethis.com/manifesto/67.04.Econosphere/pdf/ 67.04.Econosphere.pdf>, consulté le 13 novembre 2014.

22. Il s'agit d'un programme proposé par la Shannon School of Business de Cape Breton University. Cette explication est fournie en ligne par l'un des enseignants, Robert A. Campbell, spécialiste de gestion du changement et d'organisation des entreprises.

<https://managementprofessor.wordpress.com/2015/01/08/satisficing/>, consulté le 15 janvier 2015.

23. Créé par Ray Noorda, fondateur de l'entreprise de logiciel de réseaux Novell, ce terme-valise a été repris et rendu célèbre par Adam M. Brandenburger (Harvard Business School) et Barry J. Nalebuff (Yale School of Management).

24. Fonds ou actionnaires qui cherchent à faire fructifier au maximum leurs investissements 25. <http://banktivist.com/>, consulté le 10 juillet 2014. 


\section{RÉSUMÉS}

Cette étude, qui s'intéresse aux domaines de l'économie, de la gestion et de la finance, a pour objet de montrer l'intérêt qu'il y a à prendre en compte les phénomènes d'amalgames, et particulièrement les termes-valises, dans l'analyse des discours spécialisés. Après l'exposé du cadre théorique et de différentes approches des mots-valises, le choix d'une définition minimale sur laquelle fonder l'analyse est expliqué. Les quelque cinquante entrées extraites d'un large corpus de textes spécialisés (articles de recherche, ouvrages d'économie) sont présentées à travers une analyse morphologique. La spécificité des termes-valises est ensuite mise en lumière par le biais d'une analyse sémantique, au niveau des aspects théoriques et pratiques. Une veille néologique des termes-valises est recommandée, car ils sont des marqueurs de l'évolution des idées et des pratiques.

This paper, which focuses on the fields of economics, management, and finance, aims to underline how useful it is to take blending phenomena - and more specifically terminological blends - into account when analysing specialised discourse. After establishing a theoretical framework and reviewing previous research, the article delineates a minimal definition of terminological blends in support of a systematic analysis. Then the fifty items yielded by a large corpus of research articles, books and textbooks are presented from a morphological point of view. The specificity of terminological blends pertaining to theoretical or practical aspects is highlighted through a semantic analysis. As potential landmarks in the field of evolving ideas and practices, terminological blends deserve increased attention and should be closely monitored.

\section{INDEX}

Mots-clés : anglais de spécialité, mot-valise, terme-valise, terminologie de l'économie, terminologie de la gestion, terminologie de la finance, veille néologique

Keywords : economic and financial terms, English for economics, lexical blend, management term, neonym, terminological blend

\section{AUTEUR}

\section{CATHERINE RESCHE}

Catherine Resche est professeur des universités (Paris 2 - Panthéon-Assas) et elle dirige un des axes de recherche du Centre de linguistique en Sorbonne (CeLiSo). Depuis 2014, elle est directrice de la collection « Aspects linguistiques et culturels des discours spécialisés » aux éditions Peter Lang. Ses recherches portent sur l'anglais de l'économie, de la gestion et de la finance (terminologie, néologie, analyse de discours, de genre, métaphores, mise en récit). Elle a publié en 2013 un ouvrage dans la collection Linguistic Insights (Peter Lang) : Economic Terms and Beyond: Capitalising on the Wealth of Notions. catherineresche@club-internet.fr 